\title{
Fracturas de Fémur Proximal de Alta Energía Tratadas con Reducción a Través de Mini-open y Clavo Cefalo- medular
}

\section{High Energy Proximal Femur Fractures Treated with Cephalo- medullary Nails and Open Reduction through Mini-open Approach}

Ignacio E. Villalón ${ }^{1} \quad$ Jaime Cancino $^{2} \quad$ Cristóbal Vigueras $^{3}$ Iván Contesse ${ }^{4} \quad$ Julio Piriz $^{5} \quad$ Javier del Río ${ }^{1,6}$

${ }^{1}$ Hospital Clínico Mutual de Seguridad

2 Universidad del Desarrollo

3 Universidad de Valparaíso, Chile

${ }^{4}$ Universidad Austral de Chile, Chile

${ }^{5}$ Clínica Alemana de Santiago, Chile

${ }^{6}$ Clínica Las Condes, Chile

Rev Chil Ortop Traumatol 2019;60:79-85.
Address for correspondence Ignacio Villalón, Hospital Clínico Mutual de Seguridad, (e-mail: ignaciovillalonm@gmail.com).

\section{Resumen \\ Palabras clave \\ - fractura cadera \\ - alta energía \\ - joven \\ - clavo cefalo-medular}

Objetivo Evaluar los resultados radiológicos de pacientes con fractura de fémur proximal tratados con clavo cefalo-medular y reducción abierta a través de abordaje mini-open.

Material y Métodos Estudio retrospectivo-descriptivo de una serie consecutiva de 45 pacientes ( 38 hombres, 7 mujeres, edad promedio 52 años) con fractura de cadera de alta energía tratados con clavo cefalo-medular y reducción a través de abordaje miniopen. Se registró el mecanismo del accidente y presencia de lesiones asociadas. En todos los casos se contó con radiografías preoperatorias y tomografía axial computada (TAC). Se clasificaron de acuerdo a la AO. Se registró en número de fragmentos que presentaba la fractura. Se tomaron radiografías de control en forma mensual hasta la consolidación. Se midió el ángulo cervico-diafisiario operado y contralateral.

Resultados Todos los casos fueron secundarios a un accidente de alta energía. Veintidós pacientes presentaron lesiones asociadas. El número de fragmentos promedio fue de 3 . La diferencia entre el ángulo cervico-diafisiario post operatorio de la cadera fracturada versus la cadera contralateral fue de 4,9 grados. Un paciente presentó no-unión. El tiempo promedio hasta la consolidación radiográfica fue de 3,7 meses.

Conclusión El uso de clavos cefalo-medulares asociado a reducción a través de miniopen en pacientes jóvenes con fractura de fémur proximal de alta energía, presenta buenos resultados radiológicos con bajo número de complicaciones.

Objective To evaluate radiologic results of patients with proximal femur fracture treated with cephalo-medullary nails and open reduction through mini-open approach. received

May 4, 2018

accepted

September 24, 2019
DOI https://doi.org/

10.1055/s-0039-3399543. ISSN 0716-4548.
Copyright $\odot 2019$ by Thieme Revinter

Publicações Ltda, Rio de Janeiro, Brazil
License terms

(c) (1) $\ominus$ (5) 

Keywords
- hip fracture
- high energy
- young
- cephalo-medullary nails

Materials and Methods A retrospective-descriptive study of 45 patient consecutive series ( 38 male, 7 female. Mean age 52 years old) with high energy hip fracture treated with cephalo-medullary nails and open reduction through mini-open approach. Accident mechanism and associated lesions were documented. Every case was evaluated with pre-operative radiography and CT-scan. Fractures were classified according to AO classification. Patients were radiographically controlled monthly until bone healing. Cervical-diaphysial angle was measured and compared bilaterally. Results Every case was secondary to high energy trauma. 22 patients presented associated lesions. The mean number of bone fragments was 3 . The difference between postoperative cervical-diaphysial angle versus contralateral hip was $4.9^{\circ}$. Only 1 patient presented non-union. The mean time until consolidation confirmed with radiography was 3.7 months.

Conclusion The use of cephalo-medullary nails associated to open reduction through a mini-open approach in young patients with high energy associated proximal femur fractures, presents satisfactory radiologic results with a low amount of complications.

\section{Introducción}

Las fracturas de fémur proximal presentan una incidencia elevada. En el 90\% de los casos ocurren en población geriátrica en el cual la osteoporosis juega un rol importante. ${ }^{1}$ Por otra parte, el $10 \%$ restante ocurre en población joven, en el que la fractura es secundaria a un mecanismo de alta energía como lo son accidentes de tránsito y caídas de altura. ${ }^{2,3}$ Si se consideran las fracturas pertrocantéricas y subtrocantéricas, en ese grupo de pacientes la reducción y osteosíntesis puede no tener tan buenos resultados, ya que suelen ser patrones de fractura diferentes y complejos, que pueden llevar a no-unión o mal-unión y requerir por lo tanto nuevos procedimientos quirúrgicos. $^{4,5}$ Se describe en ese grupo de pacientes tasas de no-unión y falla en la osteosíntesis que pueden llegar al $20 \%{ }^{4-9}$

En la actualidad, para el tratamiento de ese tipo de lesiones, se prefiere el uso de clavos cefalo-medulares, los cuales, en diferentes series han mostrado buenos resultados. ${ }^{6-9}$ Sin embargo, los resultados que se describen en la literatura están enfocados de mayor manera en la población geriátrica y son muy pocos los estudios en los que se describen los resultados en población joven con fractura considerada de alta energía.

El objetivo de nuestro estudio es evaluar los resultados radiológicos de pacientes con fractura de fémur proximal de alta energía (pertrocantéricas y subtrocantéricas) tratados con clavo cefalo-medular y reducción abierta a través de abordaje mini-open.

\section{Material y Métodos}

Estudio retrospectivo-descriptivo de una serie consecutiva de 45 pacientes operados entre julio de 2013 y junio de 2017 en el mismo centro hospitalario (hospital de trauma), y por el mismo equipo quirúrgico conformado por 2 cirujanos de cadera. Se incluyeron todos aquellos pacientes con fractura de fémur proximal de alta energía. Treinta y ocho hombres y siete mujeres con una edad promedio de 52 años (20-59 años) se operaron en este período. Se registró el mecanismo del accidente y presencia de lesiones asociadas. En todos los casos se contó con radiografías preoperatorias (pelvis AP - axial de cadera - fémur AP y lateral en caso de las subtrocantéricas) y/o tomografía axial computada (TAC) con reconstrucción 3D. Se clasificaron de acuerdo a la AO. Se registró en número de fragmentos que presentaba la fractura y el tiempo transcurrido entre el accidente y la cirugía.

Todos los casos se operaron con el mismo clavo céfalomedular (Gamma 3, Stryker). Para la técnica quirúrgica, el paciente se posicionó en decúbito supino. En algunos casos se usó mesa ortopédica y en otros la pierna libre en mesa Jackson. De manera rutinaria utilizamos tracción (mesa ortopédica), salvo en pacientes obesos. En todos los casos se redujo la fractura de manera directa con pinzas mediante un abordaje mini-open lateral de fémur proximal. Consideramos mini-open un abordaje de menos de $7 \mathrm{~cm}$ localizado directamente sobre la zona del foco de fractura (-Figura 1). Luego, con la fractura reducida, se puso el clavo. En ningún caso de utilizó injerto complementario. En el postoperatorio se indicó fisioterapia con descarga por 6 semanas, luego 6 semanas con carga parcial y después carga a tolerancia.

Se tomaron radiografías de control en forma mensual hasta la consolidación. Se midió el ángulo cervico-diafisiario operado y contralateral.

Se registraron las complicaciones postoperatorias. Se consideraron complicaciones las siguientes: infección, nounión y falla en la osteosíntesis. Se clasificaron de acuerdo a Clavien-Dindo modificada por Sink y col. ${ }^{10}$

\section{Resultados}

Todos los casos fueron secundarios a un accidente de alta energía (-Tabla 1). Un total de 22 pacientes (48,9\%) presentaron lesiones asociadas ( - Tabla 2 ). La clasificación de las fracturas de acuerdo a la AO se presenta en la - Tabla 3. 


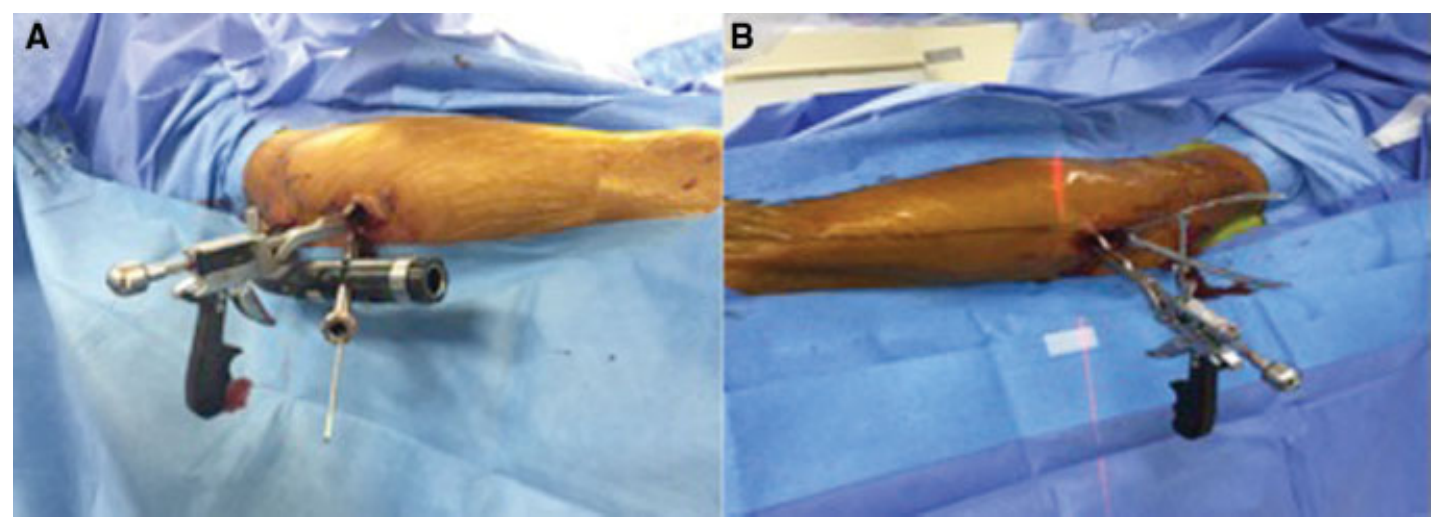

Fig. 1 Abordaje lateral al foco de fractura de no más allá $7 \mathrm{~cm}$ el cual permite reducir la fractura mediante pinzas.

Tabla 1 Mecanismo de trauma de fracturas de fémur proximal operadas

\begin{tabular}{|l|l|}
\hline Mecanismo de trauma & Número de casos \\
\hline Caída de altura (mayor a 2 metros) & 22 \\
\hline Atropello & 10 \\
\hline Accidente moto & 8 \\
\hline Caída a caballo & 3 \\
\hline Atrisión maquinaria pesada & 2 \\
\hline
\end{tabular}

Tabla 2 Lesiones asociadas de los pacientes operados

\begin{tabular}{|l|l|}
\hline Lesiones asociadas & Número de casos \\
\hline Fractura húmero & 5 \\
\hline Fractura pierna & 5 \\
\hline Fractura tobillo & 3 \\
\hline Fractura columna & 3 \\
\hline Fractura pelvis & 2 \\
\hline Trauma toraco-abdominal & 2 \\
\hline Fractura muñeca & 2 \\
\hline
\end{tabular}

Tabla 3 Clasificación AO para las fracturas de fémur proximal operadas

\begin{tabular}{|l|l|}
\hline Clasificación & Número de casos \\
\hline A1.1 & 0 \\
\hline A1.2 & 3 \\
\hline A1.3 & 3 \\
\hline A2.1 & 6 \\
\hline A2.2 & 6 \\
\hline A2.3 & 12 \\
\hline A3.1 & 9 \\
\hline A3.2 & 3 \\
\hline A3.3 & 3 \\
\hline
\end{tabular}

El número de fragmentos que presentaba la fractura fue en promedio 3 (2-7 fragmentos). El tiempo transcurrido entre el accidente y la cirugía fue de 2,4 días (1-4 días). En 6 casos $(13,3 \%)$ se utilizaron cables de acero complementarios (-Figuras 2 y 3 ).

La diferencia entre el ángulo cérvico-diafisiario postoperatorio de la cadera fracturada versus la cadera contralateral fue de $4,9^{\circ}\left(1-6^{\circ}\right)$. En total 44 pacientes $(97,8 \%)$ presentaron consolidación la cual fue en promedio a los 3,7 meses (3-5 meses). Un solo paciente (2,2\%) presentó fallo en la osteosíntesis requiriendo una nueva cirugía (grado III de acuerdo a Sink y col. ${ }^{10}$ ). Ese fue un hombre de 48 años que presentó una fractura de cadera persubtrocantérica expuesta secundaria a un atropello. En ese caso se realizó aseo quirúrgico y fijación externa el mismo día del accidente. Dos días después se realizó la osteosíntesis definitiva. Evolucionó de manera favorable hasta el quinto mes en que comenzó con dolor. La radiografía mostró fractura proximal del clavo. Se estudió posible infección y todos los parámetros fueron normales. Se realizó retiro del clavo fracturado, cruentación del foco, uso de un nuevo clavo $\mathrm{y}$ aporte de injerto óseo. Posterior a eso el paciente evolucionó bien, presentando consolidación radiológica a los 4 meses de la segunda intervención.

No se presentaron otras complicaciones.

\section{Discusión}

Las fracturas de fémur proximal en pacientes jóvenes son diferentes a la de los ancianos dado al mecanismo del trauma, lo cual lleva a compromiso de las partes blandas circundantes a la fractura y presencia de lesiones asociadas. En cuanto a la fractura propiamente tal, también es diferente a la de la población mayor, ya que suele tener patrones que dificultan la reducción de la manera habitual y requiere uso de diferentes estrategias. ${ }^{11}$ En nuestra serie, todos los casos fueron secundarios a un mecanismo de alta energía como accidentes vehiculares y caídas de altura (-Figuras 4 y 5 ). Es por ese motivo que encontramos un gran porcentaje de lesiones asociadas a la fractura de fémur proximal. Starr y col., ${ }^{11}$ plantean que ante un paciente con fractura de fémur proximal de alta energía, todas las estrategias que se saben 


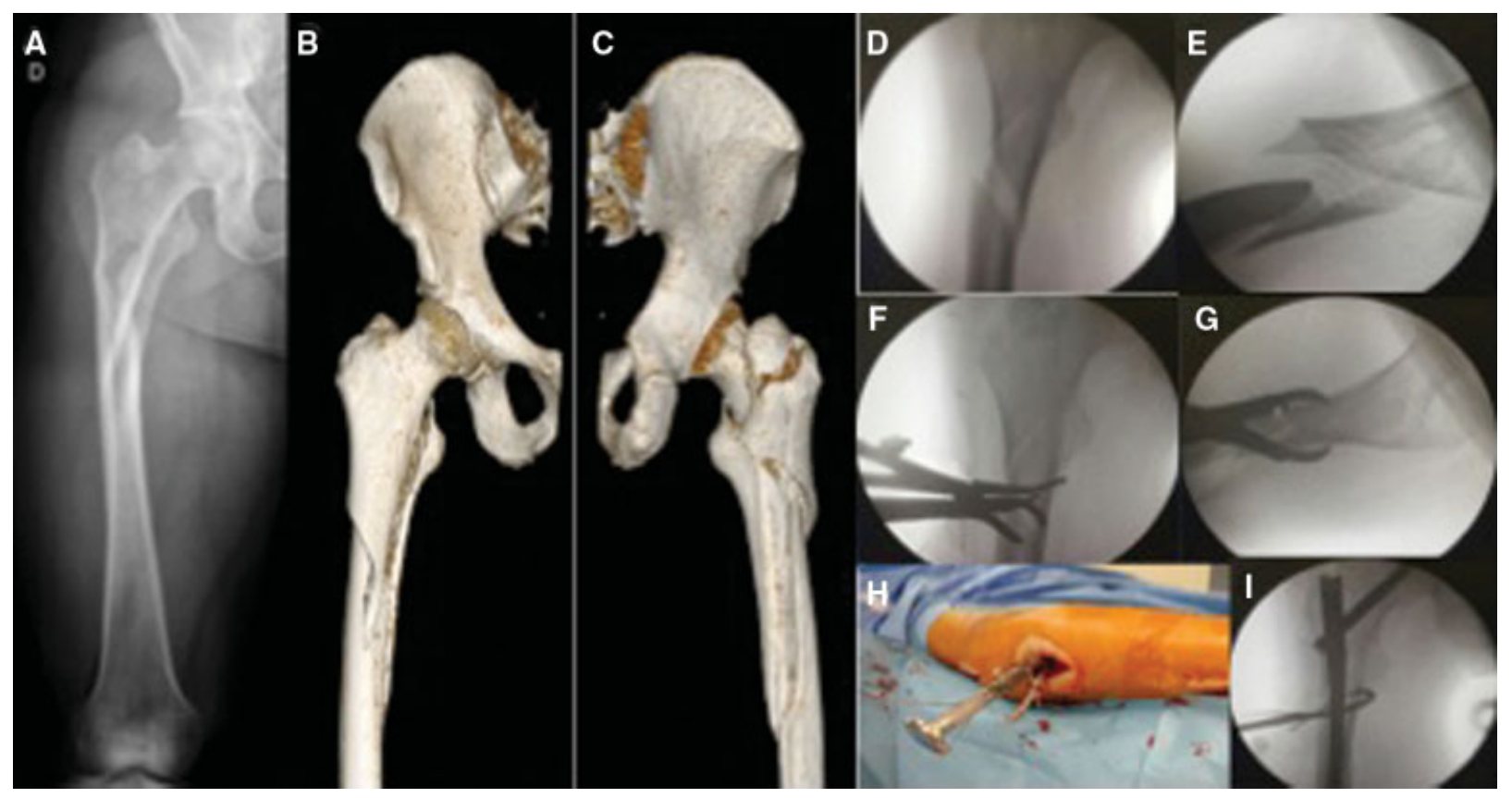

Fig. 2 Caso clínico número 1. Hombre de 47 años que sufre caída de 3 metros de altura. Radiografía de fémur (A) muestra fractura subtrocantérica de al menos 3 partes. En la TAC con reconstrucción 3-D (B y C) se muestra fractura per-subtrocantérica de 4 partes. La radioscopía intra operatoria muestra un intento frustro de reducción mediante ligamentotaxis (D y E). Mediante mini-open se permite usar pinzas para lograr reducir (F y G). Además, permite el uso de cerclajes ( $\mathbf{H}$ e I).
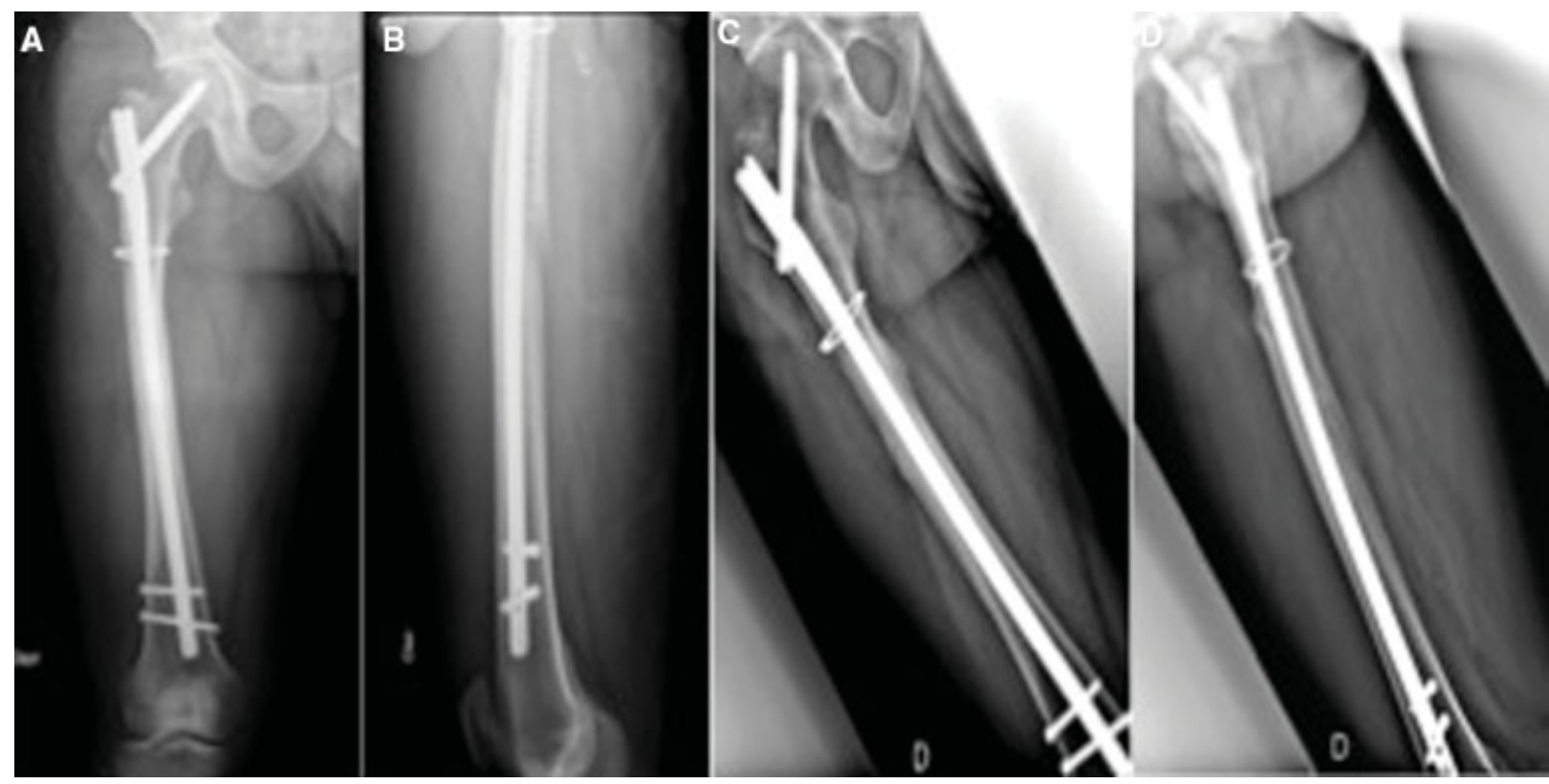

Fig. 3 Se muestran radiografías del caso clínico número 1. (A y B) radiografías de fémur AP y lateral postoperatorias inmediatas. (C y D) radiografías de fémur AP y lateral a los 4 meses consolidado.

para el manejo de fracturas en población mayor no son aplicables para ese grupo. Por lo tanto, en la gran mayoría de los casos, uno debe abrir el foco de la fractura como primer paso, independiente del elemento de osteosíntesis que utilicemos. La ligamentotaxis bajo tracción axial, muy útil en pacientes geriátricos como herramienta de reducción, no es efectiva en fracturas de cadera de alta energía. Por eso es que en ese grupo de pacientes utilizamos el mini-open de entrada para la instalación de pinzas que ayudan a reducir la fractura.
Se describen en la literatura para el manejo de esas lesiones diferentes opciones de osteosíntesis, como lo son los clavos céfalo-medulares, placas con tornillo cefálico o clavoplaca. ${ }^{12-15}$ Para todas ellas, se describen resultados buenos en general pero con un elevado porcentaje de no-unión. Eso podría estar explicado por el compromiso de partes blandas por el trauma inicial y la apertura del foco de la fractura. Hoy en día, el material de osteosíntesis de elección serían los clavos céfalomedulares, los cuales tendrían menor incidencia de no-unión o 


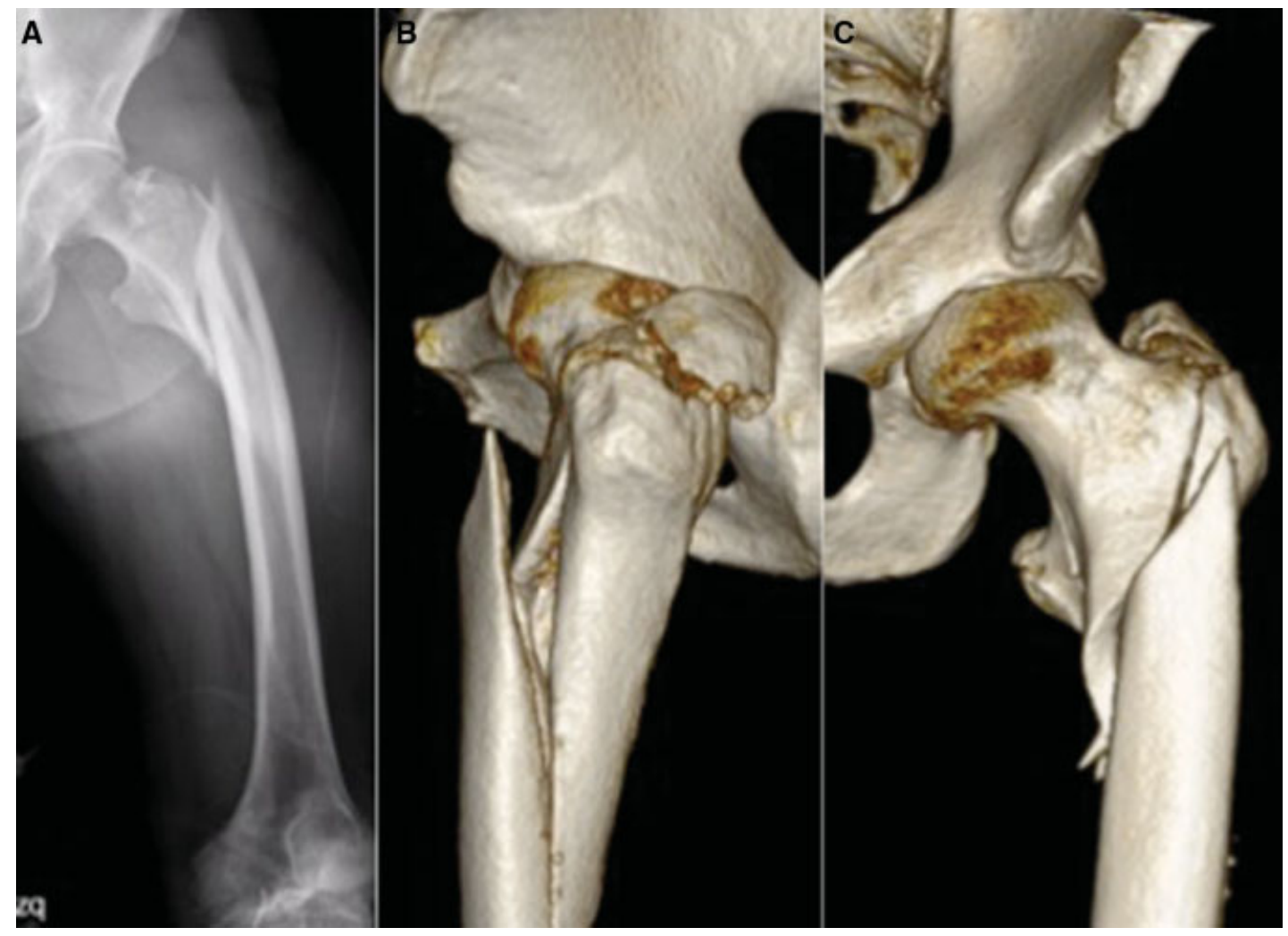

Fig. 4 Caso clínico número 2. Hombre de 46 años que sufre atropello. Radiografía muestra fractura per-subtrocantérica. TAC con reconstrucción 3-D (B y C) muestra fractura de fémur proximal en 4 partes.

mal-unión dado a las ventajas biológicas y mecánicas que tienen, las que incluyen la menor injuria de la vascularización en el sitio de la fractura y mayor soporte de stress y peso que los sistemas extra-medulares. ${ }^{16-19}$

No está establecido cuál tipo de clavo céfalo-medular según su punto de entrada es mejor para el tratamiento de pacientes con fractura de fémur proximal. Por un lado, los de entrada trocantérica podrían dañar el glúteo medio en su inserción al trocánter mayor y resecan mayor cantidad de hueso proximal. Por otro lado, en los de entrada en la fosa piriforme, esa puede ser difícil de encontrar sobre todo en pacientes obesos. ${ }^{20,21} \mathrm{La}$ entrada trocantérica suele ser más fácil de encontrar y permite realizar incisiones más pequeñas, por lo cual la elegimos para las fracturas per-subtrocantéricas. En fracturas subtrocantéricas más bajas el clavo de entrada en la fosa piriforme puede tener ventajas al facilitar la correcta alineación del fémur. Lo más importante es lograr una buena reducción con el fin de evitar el varo y el consiguiente fallo del implante. En la mayoría de los pacientes de nuestro grupo eso sólo se puede lograr reduciendo la fractura de manera directa.

Al elegir un clavo de entrada trocantérica el punto de entrada es clave. Estudios anatómicos han demostrado que para la mayoría de los pacientes la entrada debe ser en la punta del trocánter o ligeramente medial a ésta. ${ }^{22}$ Una entrada mas lateral produce varo en el fémur proximal, aumentando las posibilidades de fracaso de la osteosíntesis.

De nuestros pacientes, un 13,3\% requirió de manera adicional uso de cerclaje para obtener una adecuada reducción de la fractura. Eso no lo hacemos de manera regular, ya que esa técnica puede presentar complicaciones. ${ }^{23,24}$ Hoskins y col., ${ }^{24}$ en un estudio retrospectivo de 135 pacientes con fractura subtrocantérica cadera describen el uso de cerclaje en un $14,8 \%$. Concluyen que el uso de ese dispositivo es crucial para obtener la reducción anatómica en aquellos casos en que la reducción indirecta no lo logra. En nuestra experiencia, utilizamos el cerclaje principalmente en 2 escenarios: en aquellos pacientes con un tercer fragmento posteromedial que tras la colocación del clavo céfalo-medular se encuentra libre y en aquellos pacientes con un rasgo tipo oblicuo inverso para controlar y reducir el varo del fragmento proximal.

La diferencia del ángulo cérvico-diafisiario operado fue en promedio $4,9^{\circ}$ menor que el de la cadera no operada. Eso no influyó en la consolidación. Parry y col., ${ }^{25}$ miden el ángulo cérvico-diafisiario en caderas operadas por fractura pertrocantérica y no operadas. Concluyen que un ángulo menor al nativo se asocia de mayor manera con reducción en varo, sin embargo, no afecta el cut-out del tornillo cefálico. Ese grado de varo residual parece tolerable, ya que $44 / 45$ de nuestros pacientes consolidaron. Durante la cirugía, con la adecuada la elección del implante, punto de entrada en el trocánter y el abordaje mini-open buscamos obtener la mejor reducción postoperatoria posible (con la mínima morbilidad) y el menor varo postoperatorio.

Son pocos los estudios en la literatura de fracturas de fémur proximal de cadera de alta energía, sin embargo, en ellos se observa varo residual y no unión en alrededor del $20 \%$ de los casos. $^{11}$ En nuestra serie, todos los pacientes presentaron consolidación radiológica antes del quinto 


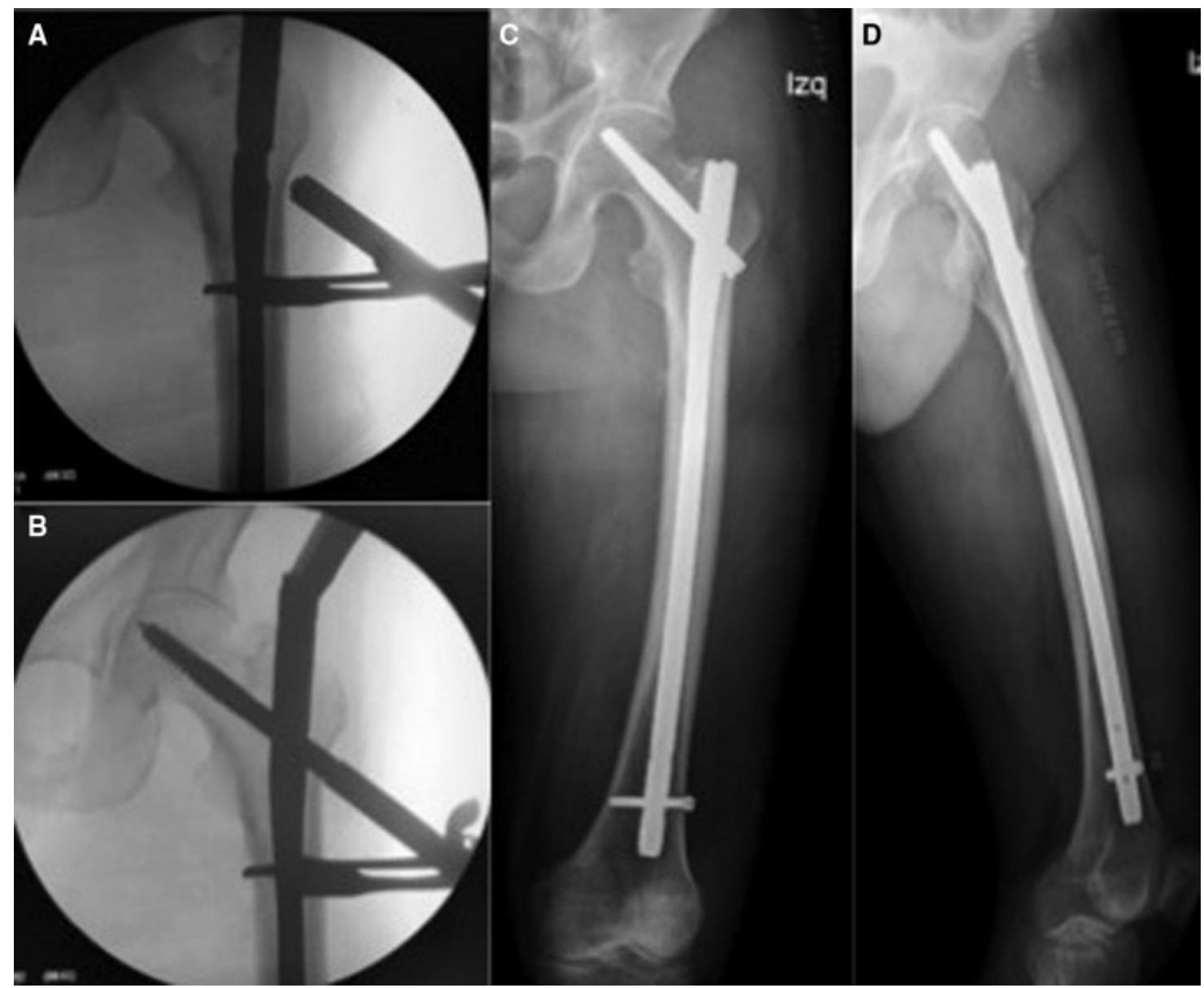

Fig. 5 Radioscopía (A y B) y radiografía postoperatoria del caso clínico número 2.

mes, excepto el paciente de la fractura expuesta. Creemos que eso se debe a la reducción inicial que se obtuvo mediante el mini-open y a la correcta elección del punto de entrada. ${ }^{22}$

En cuanto a las complicaciones, presentamos un solo caso que fue una no-unión secundaria a la falla del implante. Ese caso correspondió a una fractura expuesta de cadera en la que el clavo se fracturó al quinto mes. La reducción inicial que se obtuvo fue buena, sin embargo, creemos que podría haberse utilizado un cerclaje con el fin de aportarle mayor estabilidad a la fractura. Las fracturas expuestas de cadera son poco frecuentes $^{27}$ y probablemente ese subtipo de pacientes requiera procedimientos adicionales (dinamización del clavo, injerto), si se observa un retardo de consolidación para evitar la rotura del implante y el consiguiente fracaso de la osteosíntesis.

\section{Conclusión}

El uso de clavos céfalo-medulares asociado a reducción a través de mini-open puede ser una herramienta técnica de utilidad en el manejo de fracturas de fémur proximal de alta energía, presentando en esta serie bajas complicaciones.

\section{Conflicto de Intereses}

Lo autor del trabajo declara no tener ningún conflicto de intereses.

\section{Bibliografía}

1 Pascarella R, Fantasia R, Maresca A, et al. How evolution of the nailing system improves results and reduces orthopedic complications: more than 2000 cases of trochanteric fractures treated with the Gamma Nail System. Musculoskelet Surg 2016; 100(01):1-8

2 Bedi A, Toan Le T. Subtrochanteric femur fractures. Orthop Clin North Am 2004;35(04):473-483

3 Fielding JW, Cochran GV, Zickel RE. Biomechanical characteristics and surgical management of subtrochanteric fractures. Orthop Clin North Am 1974;5(03):629-650

4 Froimson AI. Treatment of comminuted subtrochanteric fractures of the femur. Surg Gynecol Obstet 1970;131(03):465-472

5 Saini P, Kumar R, Shekhawat V, Joshi N, Bansal M, Kumar S. Biological fixation of comminuted subtrochanteric fractures with proximal femur locking compression plate. Injury 2013;44 (02):226-231

6 Kim JW, Chang JS, Lee H, Bae JY, Kim JJ. Clinical results of femoral subtrochanteric fractures. J Korean Hip Soc 2010; 22:222-226

7 Hotz TK, Zellweger R, Kach KP. Minimal invasive treatment of proximal femur fractures with the long gamma nail: indication, technique, results. J Trauma 1999;47(05):942-945

8 Ruff ME, Lubbers LM. Treatment of subtrochanteric fractures with a sliding screw-plate device. J Trauma 1986;26(01):75-80

9 Lee JY, Lee SY. Treatment of the proximal femoral extracapsular fracture with proximal femoral nail antirotation (PFNA): Comparison with proximal femoral nail (PFN). J Korean Hip Soc 2009;19:183-189 
10 Sink EL, Leunig M, Zaltz I, Gilbert JC, Clohisy J; Academic Network for Conservational Hip Outcomes Research Group. Reliability of a complication classification system for orthopaedic surgery. Clin Orthop Relat Res 2012;470(08):2220-2226

11 Starr AJ, Hay MT, Reinert CM, Borer DS, Christensen KC. Cephalomedullary nails in the treatment of high-energy proximal femur fractures in young patients: a prospective, randomized comparison of trochanteric versus piriformis fossa entry portal. J Orthop Trauma 2006;20(04):240-246

12 Pai $\mathrm{CH}$. Dynamic condylar screw for subtrochanteric femur fractures with greater trochanteric extension. J Orthop Trauma 1996;10(05):317-322

13 Sanders R, Regazzoni P. Treatment of subtrochanteric femur fractures using the dynamic condylar screw. J Orthop Trauma 1989;3(03):206-213

14 Bridle SH, Patel AD, Bircher M, Calvert PT. Fixation of intertrochanteric fractures of the femur. A randomised prospective comparison of the gamma nail and the dynamic hip screw. J Bone Joint Surg Br 1991;73(02):330-334

15 Waddell JP. Subtrochanteric fractures of the femur: a review of 130 patients. J Trauma 1979;19(08):582-592

16 Jiang LS, Shen L, Dai LY. Intramedullary fixation of subtrochanteric fractures with long proximal femoral nail or long gamma nail: technical notes and preliminary results. Ann Acad Med Singapore 2007;36(10):821-826

17 Windolf J, Hollander DA, Hakimi M, Linhart W. Pitfalls and complications in the use of the proximal femoral nail. Langenbecks Arch Surg 2005;390(01):59-65

18 Tencer AF, Johnson KD, Johnston DW, Gill K. A biomechanical comparison of various methods of stabilization of subtrochanteric fractures of the femur. J Orthop Res 1984;2(03):297-305
19 Won Y, Yang KH, Kim KK, Weaver MJ, Allen EM. Amputated limb by cerclage wire of femoral diaphyseal fracture: a case report. Arch Orthop Trauma Surg 2016;136(12): 1691-1694

20 McConnell T, Tornetta P III, Benson E, Manuel J. Gluteus medius tendon injury during reaming for gamma nail insertion. Clin Orthop Relat Res 2003;(407):199-202

21 Georgiadis GM, Olexa TA, Ebraheim NA. Entry sites for antegrade femoral nailing. Clin Orthop Relat Res 1996;(330): 281-287

22 Ostrum RF, Marcantonio A, Marburger R. A critical analysis of the eccentric starting point for trochanteric intramedullary femoral nailing. J Orthop Trauma 2005;19(10):681-686

23 Ehlinger M, Niglis L, Favreau H, et al. Vascular complication after percutaneous femoral cerclage wire. Orthop Traumatol Surg Res 2018;104(03):377-381

24 Codesido P, Mejía A, Riego J, Ojeda-Thies C. Cerclage wiring through a mini-open approach to assist reduction of subtrochanteric fractures treated with cephalomedullary fixation: surgical technique.J J Orthop Trauma 2017;31(08):e263-e268

25 Hoskins W, Bingham R, Joseph S, et al. Subtrochanteric fracture: the effect of cerclage wire on fracture reduction and outcome. Injury 2015;46(10):1992-1995

26 Parry JA, Barrett I, Schoch B, Yuan B, Cass J, Cross W. Does the angle of the nail matter for pertrochanteric fracture reduction? Matching nail angle and native neck - shaft angle. J Orthop Trauma 2018;32(04):174-177

27 Mack AW, Freedman BA, Groth AT, Kirk KL, Keeling JJ, Andersen RC. Treatment of open proximal femoral fractures sustained in combat. J Bone Joint Surg Am 2013;95(03):e13, $1-8)$ 